\title{
Some Mathematical and Physical Remarks on Surreal Numbers
}

\author{
Juan Antonio Nieto \\ Facultad de Ciencias Fsico-Matemáticas de la Universidad Autónoma de Sinaloa, Culiacán, México \\ Email: niet@uas.edu.mx, janieto1@asu.edu
}

How to cite this paper: Nieto, J.A. (2016) Some Mathematical and Physical Remarks on Surreal Numbers. Journal of Modern Physics, 7, 2164-2176.

http://dx.doi.org/10.4236/jmp.2016.715188

Received: September 23, 2016

Accepted: November 21, 2016

Published: November 24, 2016

Copyright $\odot 2016$ by author and Scientific Research Publishing Inc. This work is licensed under the Creative Commons Attribution International License (CC BY 4.0).

http://creativecommons.org/licenses/by/4.0/ (c) (i) Open Access

\begin{abstract}
We make a number of observations on Conway surreal number theory which may be useful, for further developments, in both mathematics and theoretical physics. In particular, we argue that the concepts of surreal numbers and matroids can be linked. Moreover, we established a relation between the Gonshor approach on surreal numbers and tensors. We also comment about the possibility to connect surreal numbers with supersymmetry. In addition, we comment about possible relation between surreal numbers and fractal theory. Finally, we argue that the surreal structure may provide a different mathematical tool in the understanding of singularities in both high energy physics and gravitation.
\end{abstract}

\section{Keywords}

Surreal Numbers, Supersymmetry, Cosmology

\section{Introduction}

Surreal numbers are a fascinating subject in mathematics. Such numbers were invented, or discovered, by the mathematician John Horton Conway in the 70's [1] [2]. Roughly speaking, the key Conways idea is to consider a surreal number in terms of previously created dual sets $X_{L}$ and $X_{R}$. Here, $L$ stands for left and $R$ for right. One of the interesting things is that such numbers contain many well known ordered fields, including integer numbers, the dyadic rationals, the real numbers and hyperreals, among other numerical structures. Moreover, the structure of surreal numbers leads to a system where we can consider the concept of infinite number as naturally and consistently as any "ordinary" numbers.

It turns out that in contrast to the inductive Conway definition of surreal numbers, Gonshor [3] proposed in 1986 another definition which is based on a sequence of dual 
pluses and minuses $\{+,-\}$. Gonshor itself proves that his definition of surreal numbers is equivalent to the Conway definition.

In this article, we shall make a number of remarks on surreal number theory which we believe can be useful in both scenarios: mathematics and physics. In particular, we shall established a connection between surreal numbers and tensors. Secondly, we shall show that surreal numbers can be linked to matroids. Moreover, we shall argue that surreal numbers may be connected with spin structures and therefore may provide an interesting development in supersymmetry. We also comment about the possibility that surreal numbers are connected with fractal theory. Finally, we also mention that concepts of infinitely small and infinitely large in surreal numbers may provide a possible solution for singularities in both high energy physics and gravitation.

Technically, this work is organized as follows. In Section 2, we briefly review the Conway definition of surreal numbers. In Section 3, we also briefly review the Gonshor definition of a surreal number. In Section 4, we established a connection between surreal numbers and tensors. In Section 5, we comment about the possibility that surreal numbers and matroids are related. Moreover, in Section 6 we mention number of possible applications of the surreal number theory, division algebras, supersymmetry, black holes and cosmology.

\section{Conway Formalism}

Let us write a surreal number by

$$
x=\left\{X_{L} \mid X_{R}\right\}
$$

and call $X_{L}$ and $X_{R}$ the left and right sets of $x$, respectively. Conway develops the surreal numbers structure $\mathcal{S}$ from two axioms:

Axiom 1. Every surreal number corresponds to two sets $X_{L}$ and $X_{R}$ of previously created numbers, such that no member of the left set $x_{L} \in X_{L}$ is greater or equal to any member $x_{R}$ of the right set $X_{R}$.

Let us denote by the symbol $¥$ the notion of no greater or equal to. So the axiom establishes that if $x$ is a surreal number then for each $x_{L} \in X_{L}$ and $x_{R} \in X_{R}$ one has $x_{L} \ngtr x_{R}$. This is denoted by $X_{L} \ngtr X_{R}$.

Axiom 2. One number $X=\left\{X_{L} \mid X_{R}\right\}$ is less than or equal to another number $y=\left\{Y_{L} \mid Y_{R}\right\}$ if and only the two conditions $X_{L} ¥ y$ and $x \nsupseteq Y_{R}$ are satisfied.

This can be simplified by saying that $x \leq y$ if and only if $X_{L} \nsupseteq y$ and $x \nsupseteq Y_{R}$.

Observe that Conway definition relies in an inductive method; before a surreal number $x$ is introduced one needs to know the two sets $X_{L}$ and $X_{R}$ of surreal numbers. Thus, since each surreal number $x$ corresponds to two sets $X_{L}$ and $X_{R}$ of previous numbers then one wonders what do one starts on the zeroth day or 0-day? If one denotes the empty set by $\varnothing$ then one defines the zero as

$$
0=\{\varnothing \mid \varnothing\} \text {. }
$$

Using this, one finds that in the first day or 1-day one gets the numbers

$$
-1=\{\varnothing \mid 0\}, \quad\{0 \mid \varnothing\}=+1 .
$$


In the 2-day one has

$$
-2=\{\varnothing \mid 1\}, \quad-\frac{1}{2}=\{-1 \mid 0\}, \quad\{0 \mid 1\}=\frac{1}{2}, \quad\{1 \mid \varnothing\}=+2 .
$$

While in the 3-day one obtains

$$
-3=\{\varnothing \mid 2\},-\frac{3}{2}=\{-1 \mid 0\}, \quad \frac{1}{2}=\{0 \mid 1\}, \quad+2=\{2 \mid \varnothing\} .
$$

The process continues as the following theorem establishes:

Theorem 1. Suppose that the different numbers at the end of $n$-day are

$$
x_{1}<x_{2}<\cdots<x_{m} .
$$

Then the only new numbers that will be created on the $(n+1)$-day are

$$
\left\{\varnothing \mid x_{1}\right\},\left\{x_{1} \mid x_{2}\right\}, \cdots,\left\{x_{m-1} \mid x_{m}\right\},\left\{x_{m} \mid \varnothing\right\} .
$$

Furthermore, for positive numbers one has

$$
\left\{x_{m} \mid \varnothing\right\}=x_{m}+1
$$

and

$$
\left\{x_{m} \mid x_{m+1}\right\}=\frac{x_{m}+x_{m+1}}{2} .
$$

While defining

$$
-X=\left\{-X_{R} \mid-X_{L}\right\},
$$

for negative numbers one gets

$$
\left\{\varnothing \mid x_{m}\right\}=-\left(x_{m}+1\right)
$$

and

$$
\left\{-x_{m} \mid-x_{m+1}\right\}=-\frac{\left(x_{m}+x_{m+1}\right)}{2} .
$$

Thus, at the $n$-day one obtains $2^{n}+1$ numbers all of which are of form

$$
x=\frac{m}{2^{n}},
$$

where $m$ is an integer and $n$ is a natural number, $n>0$. Of course, the numbers (13) are dyadic rationals which are dense in the reals $R$. Let us recall this theorem:

Theorem 2. The set of dyadic rationals is dense in the reals $R$.

Proof:

Assume that $a<b$, with $a$ and $b$ elements of the reals $R$. By Archimedean property exist $n \in N$ such that

$$
0<\frac{1}{n}<b-a,
$$

which implies

$$
0<\frac{1}{2^{n}}<\frac{1}{n}<b-a .
$$

Thus, one has 


$$
1<2^{n} b-2^{n} a
$$

As the distance between $2^{n} b$ and $2^{n} a$ is grater than 1 , there is an integer $m$ such that

$$
2^{n} a<m<2^{n} b
$$

and therefore

$$
a<\frac{m}{2^{n}}<b
$$

So, the set of dyadic rationals are dense in $R$.

The sum and product of surreal numbers are defined as

$$
x+y=\left\{X_{L}+y, x+Y_{L} \mid X_{R}+y, x+Y_{R}\right\}
$$

and

$x y=\left\{X_{L} y+x Y_{L}-X_{L} Y_{L}, X_{R} y+x Y_{R}-X_{R} Y_{R} \mid X_{L} y+x Y_{R}-X_{L} Y_{R}, X_{R} y+x Y_{L}-X_{R} Y_{L}\right\}$.

The importance of (18) and (19) is that allow us to prove that the surreal number structure is algebraically a closed field. Moreover, through (18) and (19) it is also possible to show that the real numbers $R$ are contained in the surreals $\mathcal{S}$ (see Ref. [1] for details).

\section{Gonshor Formalism}

In 1986, Gonshor [3] introduced a different but equivalent definition of surreal numbers.

Definition 1. A surreal number is a function $\mu$ from initial segment of the ordinals into the set $\{+,-\}$.

For instance, if $\mu$ is the function so that $\mu(1)=+, \mu(2)=-, \mu(3)=-$, $\mu(4)=+$ then $\mu$ is the surreal number $(++-+)$. In the Gonshor approach the expressions (3)-(5) becomes: 1-day

$$
-1=(-), \quad(+)=+1,
$$

in the 2-day

$$
-2=(--),-\frac{1}{2}=(-+), \quad(+-)=+\frac{1}{2}, \quad(++)=+2,
$$

and 3-day

$$
\begin{aligned}
& -3=(---),-\frac{3}{2}=(--+),-\frac{3}{4}=(-+-),-\frac{1}{4}=(-++) \\
& (+--)=+\frac{1}{4},(+-+)=+\frac{3}{4},(++-)=+\frac{3}{2},(+++)=+3,
\end{aligned}
$$

respectively. Moreover, in Gonshor approach one finds the different numbers through the formula

$$
n|a|+\frac{|b|}{2}+\sum_{i=i}^{q} \frac{\left|c_{i}\right|}{2^{i+1}},
$$

where $a, b, c_{1}, \cdots, c_{q} \in\{+,-\}$ and $a \neq b$. Furthermore, one has $|+|=+$ and $|-|=-$. 
As in the case of Conway definition through (23) one gets the dyadic rationals. Observe that the values in (20), (21) and (22) are in agreement with (23). Just for clarity, let us consider the additional example:

$$
(++-+-+)=2-\frac{1}{2}+\frac{1}{4}-\frac{1}{8}+\frac{1}{16}=\frac{27}{16} .
$$

By the defining the order $x<y$ if $x(\alpha)<y(\alpha)$, where $\alpha$ is the first place where $x$ and $y$ differ and the convention $-<0<+$, it is possible to show that the Conway and Gonshor definitions of surreal numbers are equivalent (see Ref. [3] for details).

\section{Surreal Numbers and Tensors}

Let us introduce a $p$-tensor [4],

$$
t_{\mu_{1} \mu_{2} \cdots \mu_{p}}
$$

where the indices $\mu_{1}, \mu_{2}, \cdots, \mu_{p}$ run from 1 to 2 . Of course $p$ indicates the rank of $t_{\mu_{1} \mu_{2} \cdots \mu_{p}}$. In tensorial analysis, (25) is a familiar object. One arrives to a link with surreal numbers by making the identification $1 \rightarrow+$ and $2 \rightarrow-$. For instance, the tensor $t_{1121}$ in the Gonshor notation becomes

$$
t_{1121} \rightarrow t_{++-+} \rightarrow(++-+) .
$$

In terms of $t_{\mu_{1} \mu_{2} \cdots \mu_{p}}$, the expressions (18), (19) and (20) read

$$
-1=t_{2}, \quad t_{1}=+1,
$$

in the 2-day

$$
-2=t_{22},-\frac{1}{2}=t_{21}, \quad t_{12}=\frac{1}{2}, \quad t_{11}=2,
$$

and 3-day

$$
\begin{aligned}
& -3=t_{111},-\frac{3}{2}=t_{112},-\frac{3}{4}=t_{121},-\frac{1}{4}=t_{122}, \\
& t_{211}=+\frac{1}{4}, t_{212}=+\frac{3}{4}, t_{221}=+\frac{3}{2}, t_{222}=+3,
\end{aligned}
$$

respectively.

Formally, one note that there is a duality between positive and negative labels in surreal numbers. In fact, one can prove that this is general for any $n$-day. This could be anticipated because according to Conway definition (1) a surreal number can be written in terms of the dual pair left and right sets $X_{L}$ and $X_{R}$. Further, the concept of duality it is even clearer in the Gonshor definition of surreal numbers since in such a case one has a functions $\mu$ with codominio in the dual set $\{+,-\}$. In terms of the tensor $t_{\mu_{1} \mu_{2} \cdots \mu_{p}}$ in (25) such a duality can be written in the form

$$
t_{\mu_{1} \mu_{2} \cdots \mu_{p}}+(-1)^{q} \varepsilon_{\mu_{1} v_{1}} \varepsilon_{\mu_{2} v_{2}} \cdots \varepsilon_{\mu_{p} v_{p}} t^{v_{1} v_{2} \cdots v_{p}}=0,
$$

where

$$
\varepsilon_{\mu v}=\left(\begin{array}{cc}
0 & 1 \\
-1 & 0
\end{array}\right)
$$


It is interesting to observe that the 2-day corresponds to

$$
t_{\mu_{1} \mu_{2}}=2\left(\begin{array}{cc}
1 & 0 \\
0 & -1
\end{array}\right)+\frac{1}{2}\left(\begin{array}{cc}
0 & 1 \\
-1 & 0
\end{array}\right) \text {. }
$$

If one introduces the notation

$$
\eta_{\mu v}=\left(\begin{array}{cc}
1 & 0 \\
0 & -1
\end{array}\right)
$$

one discovers that (32) can be written as

$$
t_{\mu v}=2 \eta_{\mu v}+\frac{1}{2} \varepsilon_{\mu v} .
$$

It is worth mentioning that, in general any $2 \times 2$-matrix $\Omega_{\mu v}$ can be written as

$$
\Omega_{\mu v}=x \delta_{\mu v}+y \varepsilon_{\mu v}+r \eta_{\mu v}+s \lambda_{\mu v} .
$$

Here, one has

$$
\delta_{\mu v} \equiv\left(\begin{array}{ll}
1 & 0 \\
0 & 1
\end{array}\right)
$$

and

$$
\lambda_{\mu v} \equiv\left(\begin{array}{ll}
0 & 1 \\
1 & 0
\end{array}\right) .
$$

The set of matrices (31), (33), (36) and (37) determine a basis for any $2 \times 2$-matrix belonging to the set of $2 \times 2$-matrices which we denote by $M(2, R)$.

It is interesting that by setting $r=0$ and $s=0$ in (4) one gets the complex structure $\Omega_{\mu v} \rightarrow z_{\mu v}$, namely

$$
z_{\mu v}=x \delta_{\mu v}+y \varepsilon_{\mu v} .
$$

In fact, in the typical notation of a complex number (38) becomes $z=x+i y$. Observe also that when $\operatorname{det} \Omega \neq 0$ one obtains the group $G L(2, R)$ from $M(2, R)$. If one further requires that $\operatorname{det} \Omega=1$, then one gets the elements of the subgroup $S L(2, R)$. It is worth mentioning that the fundamental matrices $\delta_{\mu v}, \eta_{\mu v}, \lambda_{\mu v}$ and $\varepsilon_{\mu v}$ given in (31), (33), (36) and (37) not only form a basis for $M(2, R)$ but also determine a basis for the Clifford algebras $C(2,0)$ and $C(1,1)$. In fact, one has the isomorphisms $M(2, R) \sim C(2,0) \sim C(1,1)$. There exist a theorem that establishes that all the others higher dimensional algebras of any signature $C(a, b)$ can be constructed from the building blocks $C(2,0), C(1,1)$ and $C(0,2)$ (see Ref. [5] and references therein). So a connection of these developments with surreal numbers seems to be a promising scenario.

\section{Surreal Numbers and Matroids}

For a definition of a non-oriented matroid see Ref. [6] and for oriented matroid see Ref. [7] (see also Refs. [8] [9] [10] [11] [12] and references therein). Here, we shall focus in some particular cases of oriented matroids. First, assume that $\chi^{\mu v}$ satisfies the 
Grassmann-Plücker relation

$$
\chi^{\mu[v} \chi^{\alpha \beta]}=0
$$

Here, the bracket $[v \alpha \beta]$ means completely antisymmetric. In this case, the ground set of a 2-rank oriented matroid $M=\left(E, \chi^{\mu v}\right)$ is

$$
E=\{1,2,3,4\},
$$

and the alternating map becomes

$$
\chi^{\mu \nu} \rightarrow\{-1,0,1\} .
$$

The $\chi^{\mu v}$ function can be identified with a 2-rank chirotope. The collection of bases for this oriented matroid is

$$
\mathcal{B}=\{\{1,2\},\{1,3\},\{1,4\},\{2,3\},\{2,4\},\{3,4\}\},
$$

which can be obtained by just given values to the indices $\mu$ and $v$ in $\chi^{\mu v}$. Actually, the pair $(E, \mathcal{B})$ determines a 2-rank uniform non-oriented ordinary matroid.

Let us consider the underlying ground bitset (from bit and set) [13] [14]

$$
\mathcal{E}=\{1,2\}
$$

and the pre-ground set

$$
E_{0}=\{(1,1),(1,2),(2,1),(2,2)\} .
$$

One finds a relation between $E_{0}$ and $E$ by comparing (40) and (44). In fact, one has

$$
\begin{aligned}
& (1,1) \leftrightarrow 1, \quad(1,2) \leftrightarrow 2, \\
& (2,1) \leftrightarrow 3, \quad(2,2) \leftrightarrow 4
\end{aligned}
$$

This can be understood considering that (45) is equivalence relation by making the identification of indices $\{a, b\} \leftrightarrow \mu$,..., etc. Observe that considering this identifications the family of bases (42) becomes

$$
\begin{aligned}
\mathcal{B}_{0}=\{\{(1,1),(1,2)\},\{(1,1),(2,1)\},\{(1,1),(2,2)\}, \\
\\
\{(1,2),(2,1)\},\{(1,2),(2,2)\},\{(2,1),(2,2)\}\} .
\end{aligned}
$$

It turns out that the chiritope $\chi^{\mu v}$ can be associated with a 2-qubit system. So the pair $\left(E_{0}, B_{0}\right)$ can be identified with a qubitoid (a combination of qubit and matroid).

The procedure can be generalized to higher dimensions. For instance, consider the pre-ground set

$$
\begin{aligned}
& E_{0}=\{(1,1,1,1),(1,1,1,2),(1,1,2,1),(1,1,2,2), \\
&(1,2,1,1),(1,2,1,2),(1,2,2,1),(1,2,2,2) \\
&(2,1,1,1),(2,1,1,2),(2,1,2,1),(2,1,2,2) \\
&(2,2,1,1),(2,2,1,2),(2,2,2,1),(2,2,2,2)\} .
\end{aligned}
$$

It is not difficult to see that by making the identifications 


$$
\begin{array}{lll}
(1,1,1,1) \leftrightarrow \mathbf{1} & (1,1,1,2) \leftrightarrow \mathbf{2} & (1,1,2,1) \leftrightarrow \mathbf{3} \\
(1,1,2,2) \leftrightarrow \mathbf{4} & (1,2,1,1) \leftrightarrow \mathbf{5} & (1,2,1,2) \leftrightarrow \mathbf{6} \\
(1,2,2,1) \leftrightarrow \mathbf{7} & (1,2,2,2) \leftrightarrow \mathbf{8} & (2,1,1,1) \leftrightarrow \mathbf{9} \\
(2,1,1,2) \leftrightarrow \mathbf{1 0} & (2,1,2,1) \leftrightarrow \mathbf{1 1} & (2,1,2,2) \leftrightarrow \mathbf{1 2} \\
(2,2,1,1) \leftrightarrow \mathbf{1 3} & (2,2,1,2) \leftrightarrow \mathbf{1 4} & (2,2,2,1) \leftrightarrow \mathbf{1 5} \\
(2,2,2,2) \leftrightarrow \mathbf{1 6}, & &
\end{array}
$$

one obtains a relation between the pre-ground set $E_{0}$ given in (47) and the ground set

$$
E=\{1,2, \cdots, 15,16\} .
$$

This can be again understood by considering that (49) is equivalent to make the identification of indices $(a, b, c, d) \leftrightarrow \mu$, .. etc. It turns out that considering these relations one finds that the collection of bases $\mathcal{B}$ contains $\left(\begin{array}{c}16 \\ 2\end{array}\right)=120$ two-element subset of the 16-element set $E$, given in (49). This 2-element subset can be obtained by considering a lexicographic order of all 120 two-subsets of $\{\mathbf{1}, \mathbf{2}, \cdots, \mathbf{1 5}, \mathbf{1 6}\}$. One finds that the first terms of $\mathcal{B}_{0}$ look like

$$
\begin{aligned}
\mathcal{B}_{0}= & \{((1,1,1,1),(1,1,1,2)\},\{(1,1,1,1),(1,1,2,1)\},\{(1,1,1,1),(1,1,2,2)\}, \\
& \{(1,1,1,1),(1,2,1,1)\},\{(1,1,1,1),(1,2,1,2)\}, \cdots\} .
\end{aligned}
$$

(See Refs. [13] and [14] for details.)

The method, of course, can be extended to $2^{2 n+1}$-dimensions, $n=0,1,2, \cdots$ and can be connected to $\mathrm{N}$-qubit system. However, it is worth mentioning that the complete classification of $N$-qubit systems is a difficult, or perhaps an impossible task. In reference [15] an interesting development for characterizing a subclass of $\mathrm{N}$-qubit entanglement has been considered. An attractive aspect of this construction is that the $\mathrm{N}$-qubit entanglement can be understood in geometric terms. The idea is based on the bipartite partitions of the Hilbert space in the form $C^{2^{N}}=C^{L} \otimes C^{l}$, with $L=2^{N-n}$ and $l=2^{n}$. Such a partition allows a geometric interpretation in terms of the complex Grassmannian variety $\operatorname{Gr}(L, l)$ of $l$-planes in $C^{L}$ via the Plücker embedding. In this case, the Plucker coordinates of the Grassmannians are natural invariants of the theory.

There are a number of ways in which one can connect matroids with surreal numbers. First, one may think in the bitset given in (43) in the Gonshor form

$$
\mathcal{E}=\{1,2\} \rightarrow\{-,+\} .
$$

Second, the numbers of any the ground set in matroid theory

$$
E=\{1,2,3, \cdots\},
$$

can be written in terms of the surreal numbers as

$$
\{\{0, \varnothing\},\{1, \varnothing\},\{2, \varnothing\}, \cdots\} .
$$

In this context the basis set $\mathcal{B}$ will be also written in terms of the surreal numbers. Third, another possibility is also to identify the chirotope map $\chi \rightarrow\{-1,0,1\}$ in terms of the surreal numbers $\chi \rightarrow\{\{\varnothing, 0\},\{\varnothing, \varnothing\},\{0, \varnothing\}\}$. 
Of course, it will interesting to fully develop these possible links between matroids and surreal numbers. But even at these stage one note that the key concept in both matroid theory and surreal numbers theory is duality. This is because in matroid theory it is known that in matroid theory there is a key theorem that every matroid $\mathcal{M}$ has a dual $\mathcal{M}^{*}$, while in surreal number theory duality is everywhere. In a sense this is because a surreal numbers $X=\left\{X_{L}, X_{R}\right\}$ is defined in terms of two dual sets $X_{L}$ and $X_{R}$. So one wonders whether in surreal number theory exist a theorem establishing that for every surreal number set $\mathcal{S}$ there exist a dual surreal number set $\mathcal{S}^{*}$.

\section{Various Mathematical and Physical Possible Applications}

In this section we shall describe an additional number of possible applications of surreal numbers in mathematics and physics. Although such a description will be brief the main idea is to stimulate further research in the area. One may think that our proposals are in a sense for experts in the topic but in fact the main intention is to call the attention of mathematicians and physicist telling them look here are a number of subjects in which you have the opportunity to participate.

\section{Applications in mathematics:}

\section{(a) Division algebras}

There is a celebrated Hurwitz theorem:

Theorem (Hurwitz, 1898): Every normed algebra over the reals with an identity is isomorphic to one of following four algebras: the real numbers, the complex numbers, the quaternions, and the Cayley (octonion) numbers.

Moreover, the Hurwitz theorem is closely related with the parallelizable spheres $S^{1}, S^{3}$ and $S^{7}$ [16] and the remarkable theorem that only exist division algebras in 1, 2, 4 and 8 dimensions [17] [18]. So, one wonders what could be the corresponding Hurwitz theorem and these remarkable developments on division algebras if one extend the real numbers to surreal numbers. In this context, it has been proved in Refs. [19] [20] [21] that for normalized qubits the complex 1-qubit, 2-qubit and 3-qubit are deeply related to division algebras via the Hopf maps, $S^{3} \stackrel{s^{1}}{\longrightarrow} S^{2}, S^{7} \stackrel{S^{3}}{\longrightarrow} S^{4}$ and $S^{15} \stackrel{S^{7}}{\longrightarrow} S^{8}$, respectively. It seems that there does not exist a Hopf map for higher $\mathrm{N}$-qubit states. So, from the perspective of Hopf maps, and therefore of division algebras, one arrives to the conclusion that 1-qubit, 2-qubit and 3-qubit are more special than higher dimensional qubits (see Refs. [22] [23] for details). Again one wonders whether surreal numbers can contribute in this qubits theory framework.

II. Applications in physics:

(a) Supersymmetry. For finite sets $X_{L}$ and $X_{R}$, one of the key tools in surreal numbers are integers $n$ and dyadic rationals $d=\frac{m}{2^{k}}$. For $n=0,1$ and 2 and $d=\frac{1}{2}, \frac{3}{2}$ one recalls the spin structure of supersymmetry. So one wonders if for instance spin $\frac{1}{4}$ may be a prediction of surreal number theory. Remarkable, this spin has been proposed in $N=1$ supersymmetry in connection with anyons (see Refs. [24] [25] and refer- 
ences therein). Thus, for finite sets $X_{L}$ and $X_{R}$, surreal numbers $x=\left\{X_{L}, X_{R}\right\}$ and in the Gonshor approach, one finds that bosons can be identified with $s$ integer spin and fermions with dyadic rational with spin

$$
n|a|+\frac{|b|}{2}+\sum_{i=1}^{q} \frac{\left|c_{i}\right|}{2^{i+1}}
$$

given in (23). One can even think in this expression as the eigenvalues of a ket $\left|n, s_{1}, s_{2}, s_{3}, \cdots\right\rangle$. Here we made the associations

$$
s_{1} \rightarrow \frac{|b|}{2}, s_{2} \rightarrow \frac{\left|c_{1}\right|}{4}, s_{3} \rightarrow \frac{\left|c_{2}\right|}{8}, \cdots
$$

and so on. Thus, in this framework, it seems the whole structure of surreal numbers can be identified with a kind of supersymmetric approach.

(c) Black-holes

Consider the Schwarzschild metric [26]

$$
\mathrm{d} s^{2}=-\left(1-\frac{2 G M}{c^{2} r}\right) \mathrm{d} t^{2}+\frac{\mathrm{d} r^{2}}{\left(1-\frac{2 G M}{c^{2} r}\right)}+r^{2}\left(\mathrm{~d} \theta^{2}+\sin ^{2} \theta \mathrm{d} \phi^{2}\right),
$$

where $M$ is the source mass, $G$ is the Newton gravitational constant and $c$ is the light velocity. There are a number of observations that one can make about (55). First, notice that in this expression all quantities are real numbers. Second there are two type of singularities, namely in $r=r_{s}=\frac{2 G M}{c^{2}}$ and $r=0$. It is known that using Kruskal coordinates it is possible to show that the singularity at $r=r_{s}$ is simply a coordinate singularity. However the singularity at $r=0$ is a true physical singularity of spacetime. First of all, in this context, when one referes about singularity in terms of real numbers one means that in the limit $r \rightarrow 0$ one obtains the expression $\frac{2 G M}{c^{2} r} \rightarrow \infty$ (see Ref. [26] and references therein for details).

From the point of view of surreal numbers theory the singularity $\frac{2 G M}{c^{2} r} \rightarrow \infty$, when $r \rightarrow 0$, is not a real problem because in such a mathematical theory all kind of infinite large and infinite small are present. So by a assuming that all quantities in the line element given in (55) is written in terms of surreal numbers

$$
\mathrm{d} \mathcal{S}^{2}=-\left(1-\frac{2 G \mathcal{M}}{c^{2} \mathcal{R}}\right) \mathrm{d} \mathcal{T}^{2}+\frac{\mathrm{d} \mathcal{R}^{2}}{\left(1-\frac{2 G \mathcal{M}}{c^{2} \mathcal{R}}\right)}+\mathcal{R}^{2}\left(\mathrm{~d} \theta^{2}+\sin ^{2} \theta \mathrm{d} \phi^{2}\right),
$$

the problem of singularities in black-hole physics no longer exist!

(d) Cosmology

In the Friedmann cosmological equation [26]

$$
\frac{1}{a^{2}} \frac{\mathrm{d} a(t)}{\mathrm{d} t}+\frac{k}{a^{2}}-\frac{8 \pi \rho_{m}}{3}-\frac{8 \pi \rho_{r}}{3}=0,
$$

one assumes that the matter density is given by 


$$
\rho_{m}=\frac{\rho_{0 m}}{a^{3}},
$$

while the radiation energy density is

$$
\rho_{r}=\frac{\rho_{0 r}}{a^{4}},
$$

where $k, \rho_{0 m}$ and $\rho_{0 r}$ are constants. So, even if one does not consider the solution of (57) the expressions (58) and (59) tell us that there is a 'big-bang' singularity at $a \rightarrow 0$. In fact, when $a \rightarrow 0$ one has

$$
\rho_{m} \rightarrow \infty
$$

and

$$
\rho_{r} \rightarrow \infty \text {. }
$$

Just as in the case of black-holes these singularities are related to the fact that one is considering real numbers structure in the length scale $a$ as well as in the time evolution parameter $t$. Again, one wonders what formalism one may obtain by replacing a by some kind of surreal length scale $\mathcal{A}$ and the time parameter $t$ by a surreal time parameter $\mathcal{T}$. Of course, this in turn will imply that the whole gravitational theory must be modified with surreal numbers structure.

Another possibility is to identify the whole evolution of the surreal numbers structure with a cosmological model in the sense that in 0-day one has the scalar field particle of 0 -spin (the Higgs field?), in the 1-day one has (-1) -spin and 1-spin (the photon?) and the 2-day one obtains the (-2) -spin, $\left(-\frac{1}{2}\right)$-spin, $\frac{1}{2}$-spin 2-spin (graviton and fermion?) and so on. Following this idea one may even identify the 0-day and 0 -spin with the big bang and since $0=\{\varnothing, \varnothing\}$ one can say that everything in our universe started with vacuum state $\varnothing$.

\section{(e) Fractals}

It is known that fractals and dyadic fractions are deeply related. Much of this relationship can be explained by infinite binary tree which can be viewed as a certain subset of the modular group $\operatorname{PSL}(2, Z)$ (the general linear group of 2 by 2 matrices over the integers). The subset is essentially the dyadic grupoid or dyadic monoid. This in turn provides the natural setting for the symmetry and self-similarity of many fractals. Moreover, it is also that these groups and the rational numbers can be connected with dyadic subsets [27].

\section{Final Remarks}

Due to the fact that duality is the underlying concept in both surreal numbers and matroid theory, we believe that it is a matter of time that these two mathematical scenarios are considered as important tools in physics and in particular in high energy physics and gravity.

From the serious difficulties with infinities in black-hole physics and cosmology as well as in higher energy physics it seems to us that surreal numbers theory offers a new 
view for a solution, instead of thinking that the infinities are the enemies in quantum and classical physical theory incorporate them in a natural way as surreal numbers framework suggests.

It turns out that surreal numbers can be understood as a particular case of games [2] (see also Ref. [28]) which is a fascinating mathematical theory. In fact, games can be added and substracted forming an Abelian group and a sub-group of games is identified with surreal numbers which can also be multiplied and form a field. As we mentioned before, this field contains the real numbers among many other numbers structures. The key additional condition for reducing a game $x=\left\{x_{L}, \cdots, x_{R}, \cdots\right\}$ to a surreal number is that $x_{L}$ and $x_{R}$ are surreal numbers and satisfy $x_{L}<x_{R}$. So, one wonders whether game theory may lead to even more interesting applications that those presented in this work.

Finally, we believe that it is just a matter of time for the recognition of the surreal numbers structure as one of the key mathematical tools in superstring theory [29] [30] [31]. This is because although the problems of some infinities are solved there remain always additional problems with the emergency of new infinities. This phenomena may be traced back to the fact that the action in superstring theory is written in terms of real functions (target space-time coordinates) rather that surreal functions.

\section{Acknowledgements}

I would like to thank to P. A. Nieto, C. Garca-Quintero and A. Meza for helpful comments. This work was partially supported by PROFAPI/2013.

\section{References}

[1] Knuth, D.E. (1974) Surreal Numbers: How Two Ex-Students Turned on to Pure Mathematics and Found Total Happiness: A Mathematical Novelette. Addison-Wesley Publishing Co., Massachusetts.

[2] Conway, J.H. (1976) On Number and Games. London Mathematical Society Monographs, Academic Press, London.

[3] Gonshor, H. (1986) An Introduction to the Theory of Surreal Numbers. London Mathematical Society Lectures Notes Series: 110, Cambridge University Press, Cambridge.

[4] Sokolnikoff, I.S. (1951) Tensor Analysis. Chapman \& Hall, New York.

[5] Atiyah, M.F., Bott, R. and Shapiro, A. (1964) Topology, 3, 3. https:/doi.org/10.1016/0040-9383(64)90003-5

[6] Oxley, J.G. (1992) Matroid Theory. Oxford Science Publications. Oxford University Press, New York.

[7] Björner, A., Las Vergnas, M., Sturmfels, B., White, N. and Ziegler, G.M. (1993) Oriented Matroids. Cambridge University Press, Cambridge.

[8] Nieto, J.A. (2004) Advances in Theoretical and Mathematical Physics, 8, 177. arXiv: hep-th/0310071.

[9] Nieto, J.A. (2006) Advances in Theoretical and Mathematical Physics, 10, 747. arXiv: hep-th/0506106.

[10] Nieto, J.A. (2004) Journal of Mathematical Physics, 45, 285. arXiv: hep-th/0212100. 
https:/doi.org/10.1063/1.1625416

[11] Nieto, J.A. (2014) Nuclear Physics B, 883, 350-372. arXiv:1402.6998 [hep-th]. https:/doi.org/10.1016/j.nuclphysb.2014.04.001

[12] Nieto, J.A. and Marin, M.C. (2000) Journal of Mathematical Physics, 41, 7997-8005. hep-th/0005117. https:/doi.org/10.1063/1.1319518

[13] Nieto, J.A. (2013) Physics Letters B, 718, 1543-1547. arXiv:1210.0928 [hep-th]. https:/doi.org/10.1016/j.physletb.2012.12.034

[14] Nieto, J.A. (2010) Physics Letters B, 692, 43-46. e-Print: arXiv:1004.5372 [hep-th]. https:/doi.org/10.1016/j.physletb.2010.07.010

[15] Levay, P. (2005) Journal of Physics A, 38, 9075-9085. https:/doi.org/10.1088/0305-4470/38/41/016

[16] Adams, J.F. (1962) Annals of Mathematics, 75, 603-632. https:/doi.org/10.2307/1970213

[17] Bott, R. and Milnor, J. (1958) Bulletin of the American Mathematical Society, 64, 87-89. https:/doi.org/10.1090/S0002-9904-1958-10166-4

[18] Kerbvair, M. (1958) Proceedings of the National Academy of Sciences of the United States of America, 44, 286.

[19] Mosseri, R. and Dandoloff, R. (2001) Journal of Physics A, 34, 10243-10252. https:/doi.org/10.1088/0305-4470/34/47/324

[20] Mosseri, R. (2003) Two and Three Qubits Geometry and Hopf Fibrations. arXiv:quant-ph/0310053. https:/doi.org/10.1103/PhysRevD.75.024024

[21] Bernevig, B.A. and Chen, H.D. (2003) Journal of Physics A, 36, 8325-8339. https:/doi.org/10.1088/0305-4470/36/30/309

[22] Levay, P. (2007) Physical Review D, 75, Article ID: 024024. e-Print: hep-th/0610314. https:/doi.org/10.1103/PhysRevD.75.024024

[23] Levay, P. and Szalay, S. (2011) Physical Review D, 83, Article ID: 045005. e-Print: arXiv:1011.4180 [hep-th]. https:/doi.org/10.1103/PhysRevD.83.045005

[24] Mezincescu, L. and Townsend, P.K. (2010) Physical Review Letters, 105, Article ID: 191601. arXiv:1008.2334 [hep-th]. https:/doi.org/10.1103/PhysRevLett.105.191601

[25] Soroking, D. (2002) Fortschritte der Physik, 50, 724-728. https:/doi.org/10.1002/1521-3978(200205)50:5/7<724::AID-PROP724>3.0.CO;2-I

[26] Misner, C.W., Thorne, K.S. and Wheeler, J.A. (1973) Gravitation. W.H. Freeman, San Francisco.

[27] Vepstas, L. (2004) The Minkowski Question Mark, GL(2,Z) and the Modular Group. http://www.linas.org/math/chap-minkowski.pdf

[28] Schleicher, D. and Stoll, M. (2006) Moscow Mathematical Journal, 6, 359-388. arXiv:math/0410026 [math.CO].

[29] Nambu, Y. (1970) Duality and Hydrodynamics. Lectures at the Copenhagen Conference.

[30] Goto, T. (1971) Progress of Theoretical Physics, 46, 1560-1569. https:/doi.org/10.1143/PTP.46.1560

[31] Green, M., Schwarz, J. and Witten, E. (1987) Superstring Theory. Cambridge University Press, Cambridge. 
Submit or recommend next manuscript to SCIRP and we will provide best service for you:

Accepting pre-submission inquiries through Email, Facebook, LinkedIn, Twitter, etc. A wide selection of journals (inclusive of 9 subjects, more than 200 journals)

Providing 24-hour high-quality service

User-friendly online submission system

Fair and swift peer-review system

Efficient typesetting and proofreading procedure

Display of the result of downloads and visits, as well as the number of cited articles

Maximum dissemination of your research work

Submit your manuscript at: http://papersubmission.scirp.org/

Or contact jmp@scirp.org 